\title{
Liberal parentalism
}

\section{Aviad Heifetz $^{1}$ (1) | Enrico Minelli $^{2}$ | Herakles Polemarchakis ${ }^{3}$}

\author{
${ }^{1}$ Department of Management and \\ Economics, Open University of Israel, \\ Raanana, Israel \\ ${ }^{2}$ Department of Economics, University of \\ Brescia, Brescia, Italy \\ ${ }^{3}$ Department of Economics, University of \\ Warwick, Coventry, England

\section{Correspondence} \\ Aviad Heifetz, Open University of Israel, \\ Raanana, Israel. 1 University Road P.O.B. \\ 808 Ra'anana 4353701, Israel. \\ Email: aviadhe@openu.ac.il and \\ aviad.heifetz@gmail.com
}

\begin{abstract}
What normative constraints should bind parents (or policy makers) if they intervene in the choices of children (or constituencies) whose preferences evolve over time? For a sophisticated child who anticipates correctly his preference change, we prove that generically there exist parental interventions that are Pareto improving over the backward induction path that the child will follow on his own. If, in contrast, the child misperceives his future preferences, Pareto improving interventions might not exist, and even nudges might be painfully sobering. The parent may then choose to minimize the maximal disappointment along time that her benevolent intervention would cause.
\end{abstract}

\section{1 | INTRODUCTION}

Parents, in the wide sense of the term, should want their children to be happy. This is so within nuclear families, as well as between governments and their constituencies. To this effect, parents may convey to their children information that the children do not have in the first place. But after all is said, should parents, if they can, intervene in their children's choices to make them happier?

If children's preferences over action paths do not change over time, there is no such need: by the principle of optimality in dynamic programming, a child will follow from one period to the next the same overall plan that already from the very start he would like there to be implemented, and in particular no parental intervention can further enhance the child's happiness.

But what if the child's preferences over action paths do change over time? In each period, based on his current belief about his future preferences and beliefs, the child would anticipate his own future reactions ${ }^{1}$ to any choice he can make today, and then make a choice that

\footnotetext{
${ }^{1}$ Or, more generally, when the future is uncertain, the distribution of his own future reactions.
} 
together with these anticipated reactions would be optimal according to his current preferences. With a finite horizon, like when choosing education or vocational training before adulthood, or saving up to retirement, this is done, implicitly if not explicitly, by backward induction. In other words, the choices along time would constitute a Strotz-Pollak equilibrium (Goldman, 1980; Pollak, 1968; Strotz, 1955), that is, a subgame-perfect equilibrium (SPE) in the "intrapersonal game" (Laibson, 1997) among the selves of the child across the time periods.

The mature and experienced parent, in contrast, may forecast the preference evolution more accurately than the child. In addition, she may have her own perspective on how to "responsibly" average or aggregate the child's evolving preferences along time into one preference relation over action plans, so as to balance youthful vivacity with adult thriving. The optimal plan according to this aggregated preference relation may very well differ from the path that the child will follow by backward induction on his own.

So the normative question poses itself once again (see e.g., Ericson \& Laibson, 2019, open question 9): if the parent can, should she intervene and induce the optimal plan according to her overall, aggregate view of the child's evolving preferences? The question is accentuated by the fact that if the parent can intervene, nonintervention becomes one out of many possible decisions that the parent can make, and as such nonintervention need not necessarily be considered to be normatively neutral.

Some of the literature thus far (Jackson \& Yariv, 2014, 2015; Kang, 2019; Kang \& Ye, 2019; Laibson, 1997; Phelps \& Pollak, 1968) suggests that the parent should restrict herself to interventions that are Pareto-improving, that is, to inducing action paths that the child deems at least as good as the backward induction path in each and every period, and strictly better in at least one period. Such an intervention may be thought of as normatively noncontroversial, because in all periods the child will welcome such a change, enforced by the commitment power of the parent that the child himself lacks. The parent may then decide to induce the plan which is optimal according to her aggregated preferences subject to the Pareto-improvement constraint.

With this perspective, it is important to know whether Pareto-improving interventions exist in the first place. In Section 2 we therefore present a framework that makes explicit that even though bygones are bygones, current well-being may well depend also on remembered, past experience. In this framework we present a general result: when the child is sophisticated, that is, when in each period he anticipates correctly his own future preference change, then Pareto-improving interventions generically exist. That is, if for some preference evolution along time, the SPE path that a sophisticated child will follow on his own so happens to be Pareto optimal, then with slightly perturbed preferences his SPE path will no longer be Pareto optimal, and, moreover, neither will it be with further, smaller perturbations of the child's preferences. In other words, a sophisticated child chooses Pareto optimally by himself only with knife-edge profiles of preferences. ${ }^{2}$

A more realistic assumption, though, is that a child with evolving preferences is not perfectly sophisticated, but rather at least partially naive (O'Donoghue \& Rabin, 1999), that is uncertain about his own future preferences, if not outright wrong about them. In Section 3 we therefore define a simple but general type-space framework to encompass also such forms of naivité. Nudges (Thaler \& Sunstein, 2008), or more generally interventions that are purely informational, are modeled in this type-space framework by a change in the state of the world that expresses the new beliefs the child would then hold across the time periods.

\footnotetext{
${ }_{2}$ In particular, stable preferences which do not change over time is one such knife-edge case, in which by the principle of optimality a sophisticated child does
} choose Pareto optimally. 
We then show by example that with at least some naivité, even when the optimal path from the parent's perspective differs from the backward induction path, a Pareto-improving intervention might not exist, neither by inducing a different path nor by a nudge. What should the parent do in such cases?

In Section 4 we propose one possible answer, namely a normative approach by which the parent should only intervene in a way that minimizes the maximal disappointment of the child across the time periods, relative to the backward induction benchmark. We show by way of example that subject to this minimax constraint, there may indeed exist such an intervention that enhances the child's aggregated well-being from the parent's perspective.

In fact, this normative approach may be applied also to cases in which Pareto-improving interventions do exist. In these cases, the normative criterion would call the parent to choose only among interventions that maximize the minimal well-being enhancement across the time periods. We show, again by way of example, how this may (not surprisingly) alter the optimal path that the parent would induce given this additional constraint, in comparison with her intervention subject only to the Pareto-improvement constraint.

We conclude in Section 6 with a discussion. The genericity proofs and the example details appear in Appendix A.

\section{2 | SOPHISTICATED CHILDREN RARELY CHOOSE PARETO OPTIMALLY ON THEIR OWN}

For each period $i=1, \ldots, n$, let $X_{i} \subset \mathbb{R}^{k_{i}}$ be the compact, convex choice set of the child at period $i$, henceforth dubbed "self $i$." A current self cannot "preprogram" future selves' choices. ${ }^{3}$

Denote $X=X_{1} \times \cdots \times X_{n}$, and $X_{\leq i}=\prod_{j \leq i} X_{j}$ the space up-to-i initial paths. ${ }^{4}$ Let $u_{i}: X \rightarrow \mathbb{R}$ be the utility function of self $i$, where $u_{i} \in C^{\infty}(X, \mathbb{R})$, endowed with the Whitney topology. The space of utility profiles

$$
u=\left(u_{1}, \ldots, u_{n}\right) \in \mathcal{U} \equiv\left(C^{\infty}(X, \mathbb{R})\right)^{n}
$$

is endowed with the product topology. Sophistication means that with a utility profile $u$, implicitly each self $i$ anticipates correctly the future selves' utility functions $u_{i+1}, \ldots, u_{n}$, knows that each future self $j>i$ will also anticipate correctly its own future selves' utility functions $u_{j+1}, \ldots, u_{n}$, etc. ${ }^{5}$

Without intervention, a sophisticated child is assumed to choose by backward induction, that is, to follow the path $\hat{x}=\left(\hat{x}_{1}, \ldots, \hat{x}_{n}\right)$ induced by a Strotz-Pollak equilibrium of best replies $\left(b_{i}: X_{\leq i-1} \rightarrow X_{i}\right)_{i=1}^{n}$, which is a SPE of the perfect-information dynamic game between the selves. ${ }^{6}$ At such an equilibrium

\footnotetext{
${ }^{3}$ Put differently, whatever device a particular self does have, if at all, for influencing future selves' behavior, choosing to influence future behavior in such a way is encoded within the available choices $x_{i} \in X_{i}$, and by the utility functions (as defined next) of future selves $j>i$, that depend in particular on $x_{i}$. We restrict attention to a finite-horizon analysis—for the infinite-horizon case, see for example, Mertens and Rubinchik (2017), Strulik (2021).

${ }^{4}$ Or, more generally, $X \subseteq X_{1} \times \cdots \times X_{n}$ and $X_{\leq i} \subseteq \prod_{j \leq i} X_{j}$ - in case the past choice path $\left(x_{1}, \ldots, x_{j-1}\right)$ of the selves $k<j$ may limit the feasible choices of self $j$. ${ }^{5}$ In Section 3 we will present an extended framework where this assumption can be made explicit, a framework that also allows for various deviations from such perfect sophistication.

${ }^{6}$ For proofs of equilibrium existence see Harris (1985), Hellwig and Leininger (1987), Hellwig et al. (1990), Alós-Ferrer and Ritzberger (2016). If best replies are not unique, breaking ties in different ways may lead to multiple equilibria.
} 


$$
\begin{array}{ll}
b_{n}\left(x_{\leq n-1}\right) & \in \underset{X_{n}}{\arg \max _{n}\left(x_{\leq n-1}, \cdot\right)} \\
b_{n-1}\left(x_{\leq n-2}\right) & \in \arg \max _{X_{n-1}} u_{n-1}\left(x_{\leq n-2}, \cdot, b_{n}\left(x_{\leq n-2}, \cdot\right)\right) \\
b_{n-2}\left(x_{\leq n-3}\right) & \in \arg \max _{X_{n-2}} u_{n-2}\left(x_{\leq n-3}, \cdot, b_{n-1}\left(x_{\leq n-3}, \cdot\right), b_{n}\left(x_{\leq n-3}, \cdot, b_{n-1}\left(x_{\leq n-3}, \cdot\right)\right)\right) \\
& \vdots \\
b_{1}(\varnothing) & \in \arg \max _{X_{1}} u_{1}\left(\cdot, b_{2}(\cdot), b_{3}\left(\cdot, b_{2}(\cdot)\right), \ldots\right) .
\end{array}
$$

The corresponding backward induction path $\hat{x}=\left(\hat{x}_{1}, \ldots, \hat{x}_{n}\right)$ is then defined inductively by

$$
\begin{aligned}
\hat{x}_{1} & =b_{1}(\varnothing) \\
\hat{x}_{2} & =b_{2}\left(\hat{x}_{1}\right) \\
\hat{x}_{3} & =b_{3}\left(\hat{x}_{1}, \hat{x}_{2}\right) \\
& \vdots \\
\hat{x}_{n} & =b_{n}\left(\hat{x}_{1}, \hat{x}_{2}, \ldots, \hat{x}_{n-1}\right) .
\end{aligned}
$$

It yields the utility levels $\hat{u}=\left(\hat{u}_{1}, \ldots, \hat{u}_{n}\right)$ where $\hat{u}_{i}=u_{i}(\hat{x})$.

The parent has an average/aggregation function $V: \mathbb{R}^{n} \rightarrow \mathbb{R}$ over the utility levels of the different selves $i=1, \ldots, n$. This defines, indirectly, the utility function of the parent $v: X \rightarrow \mathbb{R}$ over choice paths, $v(x)=V\left(u_{1}(x), \ldots, u_{n}(x)\right)$.

For simplicity we assume that the parent can oblige the child to follow any path $x \in X$ that the parent likes. ${ }^{7}$ Still, the parent may like to limit herself to a subset of paths with some normatively desirable properties.

A path $x \in X$ is a called a Pareto improvement over a path $\bar{x} \in X$ if $u_{i}(x) \geq u_{i}(\bar{x})$ for every $i=1, \ldots, n$, and $u_{j}(x)>u_{j}(\bar{x})$ for some $1 \leq j \leq n$. Suppose, first, that the parent would like to limit herself to inducing the child only to choice paths $x$ that are Pareto improvements over the backward induction path $\hat{x}$ that the child would follow on his own.

Example 1. A sophisticated child has to finish a chore of size 3 in 3 days $i=1,2,3$. Carrying out quantity $x_{i}$ of the chore on day $i$ takes $x_{i}^{2}$ hours, and the child enjoys his remaining leisure time $24-x_{i}^{2} .^{8}$ The child's time preference is captured by $\beta-\delta$ quasihyperbolic discounting, with $\beta=\frac{1}{2}$ and $\delta=1$. At Days 2,3 the child's memory of his past leisure augments his well-being, with no discounting. Thus, if the child's division of the chore along days $i=1,2,3$ is $x=\left(x_{1}, x_{2}, x_{3}\right)$, his corresponding selves' utility functions are

$$
\begin{aligned}
& u_{1}\left(x_{1}, x_{2}, x_{3}\right)=\left(24-x_{1}^{2}\right)+\frac{1}{2}\left(24-x_{2}^{2}\right)+\frac{1}{2}\left(24-x_{3}^{2}\right) \\
& u_{2}\left(x_{1}, x_{2}, x_{3}\right)=\left(24-x_{1}^{2}\right)+\left(24-x_{2}^{2}\right)+\frac{1}{2}\left(24-x_{3}^{2}\right) \\
& u_{3}\left(x_{1}, x_{2}, x_{3}\right)=\left(24-x_{1}^{2}\right)+\left(24-x_{2}^{2}\right)+\left(24-x_{3}^{2}\right) .
\end{aligned}
$$

Across the 3 days the parent values equally the child's happiness, ${ }^{9}$ leading to the parent's utility function

\footnotetext{
$\overline{7}$ We note that leading the child to follow a particular path $x$ need not necessarily involve direct coercion by the parent. Instead, the parent may be able to provide incentives for the child to follow $x$, for example, via taxes or subsidies accompanied by an unconditional lump-sum transfer, as in Kang (2019).

${ }^{8}$ This example is similar to the "cake-eating" problem under changing tastes, as analyzed, for example, in Goldman (1979). Here, in contrast, the felicity from the good (leisure) is linear whereas the 'cost' of leisure (time put aside for the chore) is convex.
} 


$$
v\left(x_{1}, x_{2}, x_{3}\right)=\frac{1}{3} \sum_{i=1}^{3} u_{i}\left(x_{1}, x_{2}, x_{3}\right)=\left(24-x_{1}^{2}\right)+\frac{5}{6}\left(24-x_{2}^{2}\right)+\frac{2}{3}\left(24-x_{3}^{2}\right) .
$$

With the constraint $x_{3}=3-x_{1}-x_{2}$, one can verify (see the details in Appendix A) that

$$
\bar{x}=\arg \max _{x_{1}, x_{2}}\left(x_{1}, x_{2}, 3-x_{1}-x_{2}\right)
$$

is not a Pareto improvement over the backward induction path $\hat{x}$.

However, Pareto improvements over $\hat{x}$ do exist:

(a) The child would be happy to work somewhat more on Day 1 if only he could know that he will be bound to work somewhat more also on Day 2, and not leave so much of the chore to the last day, as he correctly anticipates on Day 1 that he would be doing on Day 2 without intervention;

(b) On Day 2 the child would be happy to be committed to a package deal in which he works somewhat more on both Days 1 and 2; and

(c) On Day 3 the child would definitely be happy if less of the largest share of the chore is left for him to finish.

Moreover, the Pareto improving path $\breve{x}$ that maximizes $v$ satisfies $v(\breve{x})>v(\hat{x})$, so the parent would like to intervene and induce $\breve{x}$ (even though implementing the non-Pareto improving $\bar{x}$ would entail an even higher utility, $v(\bar{x})>v(\breve{x})$ ).

A path $\tilde{x} \in X$ is called Pareto optimal if there does not exist a Pareto improvement $x \in X$ over $\tilde{x}$.

Theorem There is an open and dense subset of utility profiles $\mathcal{U}_{0} \subseteq \mathcal{U}$ of sophisticated children, for whom no interior backward induction path $\hat{x}$ is Pareto optimal.

The proof, based on a transversality argument, appears in Appendix A.

Thus, for sophisticated children with typical or generic utility profiles, namely utility profiles in $\mathcal{U}_{0}$, there exists a Pareto improving path $x \in X$ over any interior backward induction path $\hat{x}$. If the parent can induce the child to follow a Pareto improving $x$ instead of $\hat{x}$, this will be weakly preferred by the child in all time periods, and strictly preferred in some time period(s). Moreover, if the parent's utility is some weighted average of the child's utility levels at the different time periods, then also the parent will prefer to induce $x$ instead of letting the child follow the backward induction path $\hat{x}$ on his own. ${ }^{10}$

Remark 1. Dubey (1986) proved that in generic simultaneous-move games, no Nash equilibrium is Pareto optimal. Our proof is more involved, because the sequential nature of the interaction between the selves implies that a self's choice affects its utility not only directly, but also indirectly via its influence on future selves' best replies. Our proof thus applies to perfectinformation extensive form games in which every player acts once. We restricted our attention to interior equilibria, whereas Dubey (1986) addressed also equilibria on the boundary of the strategy sets; such an extension for our case awaits future investigation.

\footnotetext{
${ }^{9}$ Not the child's leisure time-that would have amounted to a complete identification of the parent with the child's perspective on Day 3 , waving off the child's perspectives on Days 1 and 2 .

${ }^{10}$ Kang and Wang (2019) proved that Pareto improvements are preferred as well by the parent also in a related but different setting, in which each self's myopic utility depends only on the present and the future, while the normative perspective of the parent is based on aggregation from the fictitious period 0 .
} 


\section{I A TYPE SPACE FOR POSSIBLE MISPERCEPTIONS ABOUT FUTURE PREFERENCES}

But children are not always sophisticated, and might anticipate their future preferences, and therefore their future choices, differently than they will actually be. Examples of such naivité or partial naivité were discussed, for example, by O'Donoghue and Rabin (1999). The following type space framework is intended to capture such states of affairs in general.

For each self $i=1, \ldots, n$, let $T_{i}$ be a measurable space of self $i$ 's types. Types $\tau_{i} \in T_{i}$ are measurably associated with

1. a belief (i.e., a probability measure) $\beta_{\tau_{i}}$ on the states of the world $T \subseteq \prod_{j=1}^{n} T_{j}$, where $\beta_{\tau_{i}}$ features perfect recall, that is, has the property that in each state $\tau=\left(\tau_{1}, \ldots, \tau_{n}\right) \in T, \beta_{\tau_{i}}\left(\left\{\left(\tau_{1}, \ldots, \tau_{i}\right)\right\} \times \prod_{j=i+1}^{n} T_{j}\right)=1 ;{ }^{11,12}$

2. a measurable utility function $u_{\tau_{i}}: X \rightarrow \mathbb{R}$, that not only represents preferences over choice paths $x \in X$, but also meaningfully expresses well-being comparably across selves, ${ }^{13,14}$ and

3. a measurable best-reply function $b_{\tau_{i}}: X_{\leq i-1} \rightarrow X_{i}$, where

$$
\begin{aligned}
& b_{\tau_{n}}\left(x_{\leq n-1}\right) \quad \in \arg \max _{X_{n}} u_{\tau_{n}}\left(x_{\leq n-1}, \cdot\right) \\
& b_{\tau_{n-1}}\left(x_{\leq n-2}\right) \in \underset{X_{n-1}}{\arg \max } \int_{T_{n}} u_{\tau_{n-1}}\left(x_{\leq n-2}, \cdot, b_{\tilde{\tau}_{n}}\left(x_{\leq n-2}, \cdot\right)\right) \mathrm{d} \beta_{\tau_{n-1}}\left(\tilde{\tau}_{n}\right) \\
& b_{\tau_{n-2}}\left(x_{\leq n-3}\right) \in \underset{X_{n-2}}{\arg \max } \int_{T_{n-1} \times T_{n}} u_{\tau_{n-2}}\left(x_{\leq n-3}, \cdot, b_{\tilde{\tau}_{n-1}}\left(x_{\leq n-3}, \cdot\right), b_{\tilde{\tau}_{n}}\left(x_{\leq n-3}, \cdot, b_{\tilde{\tau}_{n-1}}\left(x_{\leq n-3}, \cdot\right)\right)\right) \\
& \mathrm{d} \beta_{\tau_{n-2}}\left(\tilde{\tau}_{n-1}, \tilde{\tau}_{n}\right) \\
& b_{\tau_{1}}(\varnothing) \quad \in \arg \max _{X_{1}} \int_{T_{2} \times \cdots \times T_{n}} u_{\tau_{1}}\left(\cdot, b_{\tilde{\tau}_{2}}(\cdot), b_{\tilde{\tau}_{3}}\left(\cdot, b_{\tilde{\tau}_{2}}(\cdot)\right), \ldots\right) \mathrm{d} \beta_{\tau_{1}}\left(\tilde{\tau}_{2}, \ldots, \tilde{\tau}_{n}\right)
\end{aligned}
$$

for $i=n, \ldots, 1 .^{15,16}$

\footnotetext{
${ }^{11}$ So when no confusion may arise, we will interchangeably refer to $\beta_{\tau_{i}}$ as a belief on $\prod_{j=i+1}^{n} T_{j}$.

${ }^{12}$ The space of beliefs $\mu$ on a measurable space $Y$ is endowed with the $\sigma$-algebra generated by the sets of the form $\{\mu: \mu(E) \geq p\}$ for $p \in[0,1]$ and measurable events $E \subseteq Y$.

${ }^{13}$ To the latter effect, in the background there may, for example, be for each type $\tau_{j}$ of self $j$ an instantaneous felicity function $f_{\tau_{j}}: X_{j} \rightarrow \mathbb{R}$, so that

$$
u_{\tau_{i}}\left(x_{1}, \ldots, x_{n}\right)=\sum_{j=1}^{n} \omega_{\tau_{i}}(j)\left(\int_{T}\left(f_{\tau_{j}}\left(x_{j}\right)\right) d \beta_{\tau_{i}}\right)
$$
}

for some positive weights $\omega_{\tau_{i}}(j)$ (these weights may represent, for example, time discounting by the type $\tau_{i}$ ); or, more generally

$$
u_{\tau_{i}}\left(x_{1}, \ldots, x_{n}\right)=\sum_{j=1}^{n}\left(\int_{T} \int_{X_{j}} \omega_{\tau_{i}}\left(\widetilde{x}_{j} ; x_{j}\right) f_{\tau_{j}}\left(\widetilde{x}_{j}\right) d \mu_{\tau_{i}}\left(\widetilde{x}_{j} ; x_{j}\right) d \beta_{\tau_{i}}\right)
$$

where for $\tilde{x}_{j}=x_{j}$ the weight $\omega_{\tau_{i}}\left(x_{j} ; x_{j}\right)$ for experienced felicity is positive, and for $\tilde{x}_{j} \neq x_{j}$ the weight $\omega_{\tau_{i}}\left(\tilde{x}_{j} ; x_{j}\right)$ for forgone or counterfactual, unexperienced felicity is nonpositive (representing regret); and where the probability measure $\mu_{\tau_{i}}\left(\cdot x_{j}\right)$ on $X_{j}$ expresses the relative importance that $\tau_{i}$ attaches to the experienced felicity of $x_{j}$ by self $j$ versus the unexperienced, forgone felicity of other $\tilde{x}_{j} \neq x_{j}$ by self $j$.

${ }^{14}$ The space of utility functions is endowed with the Borel $\sigma$-algebra of the topology of pointwise convergence.

${ }^{15}$ We need to specify $b_{\tau_{i}}$ explicitly for each type $\tau_{i} \in T_{i}$, because $u_{\tau_{i}}$ and $\beta_{\tau_{i}}$ on their own (i) might sometimes be compatible with several best reply functions (when ties can be broken in several ways), and (ii) might sometimes be compatible with no best reply function at all, when $u_{\tau_{i}}$ has discontinuities, or when for some $j>i$ discontinuities in the best replies $b_{\tau_{j}}$ of types $\tau_{j}$ in the support of $\beta_{\tau_{i}}$ hinder the expectation of $u_{\tau_{i}}$ with respect to $\beta_{\tau_{i}}$ from attaining a maximum in $X_{i}$ (see examples of such situations in Hellwig \& Leininger, 1987 and Hellwig et al., 1990). Thus, $\tau_{i}$ being measurably associated with $\left(\beta_{\tau_{i}}, u_{\tau_{i}}, b_{\tau_{i}}\right)$ means in particular that $\beta_{\tau_{i}}, u_{\tau_{i}}, b_{\tau_{i}}$ are mutually compatible.

${ }^{16}$ The space of best-reply functions is endowed with the Borel $\sigma$-algebra of the topology of pointwise convergence. 
For (a particularly simple) example, each utility profile $u=\left(u_{1}, \ldots u_{n}\right)$ of a sophisticated child, together with a Strotz-Pollak equilibrium $\left(b_{i}: X_{\leq i-1} \rightarrow X_{i}\right)_{i=1}^{n}$ defines a type space with a single state $\tau=\left(\tau_{1}, \ldots, \tau_{n}\right)$ where $u_{\tau_{i}}=u_{i}, \beta_{\tau_{i}}(\{\tau\})=1$ and $b_{\tau_{i}}=b_{i}$ for $i=1, \ldots, n$.

In the state of the world $\tau=\left(\tau_{1}, \ldots, \tau_{n}\right)$ the backward induction path $\hat{x}_{\tau}=\left(\hat{x}_{\tau, 1}, \ldots, \hat{x}_{\tau, n}\right)$ is defined inductively by

$$
\begin{aligned}
\hat{x}_{\tau, 1} & =b_{\tau_{1}}(\varnothing) \\
\hat{x}_{\tau, 2} & =b_{\tau_{2}}\left(\hat{x}_{\tau, 1}\right) \\
\hat{x}_{\tau, 3} & =b_{\tau_{3}}\left(\hat{x}_{\tau, 1}, \hat{x}_{\tau, 2}\right) \\
& \vdots \\
\hat{x}_{\tau, n} & =b_{\tau_{n}}\left(\hat{x}_{\tau, 1}, \hat{x}_{\tau, 2}, \ldots, \hat{x}_{\tau, n-1}\right) .
\end{aligned}
$$

Since each type remembers correctly past types and choices, the (expected) well-being levels at $\tau$ under backward induction are

$$
\begin{aligned}
\hat{u}_{\tau}= & \left(\hat{u}_{\tau_{1}}, \ldots, \hat{u}_{\tau_{i}}, \ldots, \hat{u}_{\tau_{n}}\right) \\
= & \left(\int_{T_{2} \times \cdots \times T_{n}} u_{\tau_{1}}\left(\hat{x}_{\left(\tau_{1}, \tilde{\tau}_{2}, \ldots \tilde{\tau}_{n}\right)}\right) \mathrm{d} \beta_{\tau_{1}}\left(\widetilde{\tau}_{2}, \ldots, \widetilde{\tau}_{n}\right), \ldots, \int_{T_{i+1} \times \cdots \times T_{n}} u_{\tau_{i}}\left(\hat{x}_{\left(\tau_{1}, \ldots \tau_{i}, \tau_{i+1}, \ldots \tau_{n}\right)}\right) \mathrm{d} \beta_{\tau_{i}}\left(\widetilde{\tau}_{i+1}, \ldots, \widetilde{\tau}_{n}\right), \ldots,\right. \\
& \left.u_{\tau_{n}}\left(\hat{x}_{\tau}\right)\right) .
\end{aligned}
$$

We assume, for simplicity, that the parent knows the true, prevailing state of the world $\tau$, that is, the parent knows how the beliefs, preferences, and best replies of the child are about to evolve. ${ }^{17}$ We further assume that if the parent intervenes and induces a path $x \in X$, the parent is open and honest to the child from the very start about the path that the child is henceforth about to follow, ${ }^{18}$ which would therefore induce the well-being levels

$$
u_{\tau_{1}}(x), \ldots, u_{\tau_{n}}(x) .
$$

At the state of the world $\tau=\left(\tau_{1}, \ldots, \tau_{n}\right) \in T$, a path $x \in X$ that the parent may induce is a Pareto improvement over backward induction if $u_{\tau_{i}}(x) \geq \hat{u}_{\tau_{i}}$ for $i=1, \ldots, n$, and $u_{\tau_{j}}(x)>\hat{u}_{\tau_{j}}$ for some $1 \leq j \leq n$.

The state $\tau$ represents the utilities and beliefs of the child's selves after any information exchange between the parent and the child has already taken place. Ex ante information exchange, to the extent that it influences the beliefs of the child, amounts to altering the prevailing state of the world to some other state $\tau^{\prime} \in T$.

As before, the parent has an average/aggregation function $V: \mathbb{R}^{n} \rightarrow \mathbb{R}$ over the utility levels of the different selves $i=1, \ldots, n$, that the parent wants to maximize. In each state of the world $\tau=\left(\tau_{1}, \ldots, \tau_{n}\right) \in T$, this induces a utility function of the parent $v_{\tau}: X \rightarrow \mathbb{R}$ over choice paths, defined by

$$
v_{\tau}(x)=V\left(u_{\tau_{1}}(x), \ldots, u_{\tau_{n}}(x)\right)
$$

\footnotetext{
${ }^{17}$ See the discussion in Section 6 on relaxing this assumption.

${ }^{18}$ In Section 6 we will discuss an extension, where even though the parent actually knows the prevailing state of the world $\tau=\left(\tau_{1}, \ldots, \tau_{n}\right)$, she can openly and honestly announce a state-contingent policy, by which the induced action $x_{i}$ in period $i$ may depend on the realized type $\tilde{\tau}_{i}$ in that period.
} 


\section{I WITH MISPERCEPTIONS, PARETO-IMPROVING INTERVENTIONS MIGHT RESILIENTLY LACK}

For naive or partially naive types, who misperceive their future preferences, there might exist no path that all selves will at least weakly prefer over (their anticipation from) backward induction choices, with strict preference for some self. This is demonstrated in the following example.

Example 2. With the feasible action paths and utility functions specified in Example 1, consider a type space with two states of the world, $\left(\dot{\tau}_{1}, \dot{\tau}_{2}, \dot{\tau}_{3}\right)$ and $\left(\dot{\tau}_{1}, \ddot{\tau}_{2}, \ddot{\tau}_{3}\right)$, with the beliefs

$$
\begin{aligned}
& \beta_{\dot{\tau}_{1}}\left\{\left(\dot{\tau}_{1}, \ddot{\tau}_{2}, \ddot{\tau}_{3}\right)\right\}=1 \\
& \beta_{\dot{\tau}_{2}}\left\{\left(\dot{\tau}_{1}, \dot{\tau}_{2}, \dot{\tau}_{3}\right)\right\}=1, \quad \beta_{\ddot{\tau}_{2}}\left\{\left(\dot{\tau}_{1}, \ddot{\tau}_{2}, \ddot{\tau}_{3}\right)\right\}=1 \\
& \beta_{\dot{t}_{3}}\left\{\left(\dot{\tau}_{1}, \dot{\tau}_{2}, \dot{\tau}_{3}\right)\right\}=1, \quad \beta_{\ddot{t}_{3}}\left\{\left(\dot{\tau}_{1}, \ddot{\tau}_{2}, \ddot{\tau}_{3}\right)\right\}=1
\end{aligned}
$$

and the utility functions

$$
\begin{aligned}
& u_{\dot{\tau}_{1}}=u_{1} \\
& u_{\dot{\tau}_{2}}=u_{2}, \quad u_{\tilde{t}_{2}}=u_{3} \\
& u_{\dot{\tau}_{3}}=u_{\tilde{\tau}_{3}}=u_{3 .} .
\end{aligned}
$$

The best replies of the types are uniquely determined by these beliefs and utilities, and are computed in Appendix A.

In state $\left(\dot{\tau}_{1}, \dot{\tau}_{2}, \dot{\tau}_{3}\right)$ the selves have the same utility functions as in the previous example, but self 1 naively believes that as of tomorrow the present bias will miraculously disappear $\left(u_{\ddot{t}_{2}}=u_{3}\right)$; he moreover wrongly believes that this is commonly known: the state $\left(\dot{\tau}_{1}, \ddot{\tau}_{2}, \ddot{\tau}_{3}\right)$, in which $\dot{\tau}_{1}$ believes, is common knowledge at $\left(\dot{\tau}_{1}, \ddot{\tau}_{2}, \ddot{\tau}_{3}\right)$.

Moreover, in state $\left(\dot{\tau}_{1}, \ddot{\tau}_{2}, \ddot{\tau}_{3}\right)$ the selves of all three periods, $u_{\dot{\tau}_{1}}, u_{\ddot{\tau}_{2}}$ and $u_{\ddot{\tau}_{3}}$, value equally the leisure time at periods 2 and 3. Therefore, by the principle of optimality, the backward induction path $\hat{x}_{\left(\dot{\tau}_{1}, \ddot{z}_{2}, \dot{\tau}_{3}\right)}$ already maximizes $u_{i_{1}}$ over all possible paths. Since $u_{\dot{\tau}_{1}}$ is strictly concave, any other path $x \neq \hat{x}_{\left(\dot{\tau}_{1}, \dot{\tau}_{2}, \dot{\tau}_{3}\right)}$ would actually decrease $u_{\dot{t}_{1}}$, that is, $u_{\dot{t}_{1}}(x)<\hat{u}_{\dot{\tau}_{1}}$. In other words, there exists no intervention by the parent which would not decrease the well-being of $\dot{\tau}_{1}$ in the state $\left(\dot{\tau}_{1}, \ddot{\tau}_{2}, \ddot{\tau}_{3}\right)$.

But this means that also in the state $\left(\dot{\tau}_{1}, \dot{\tau}_{2}, \dot{\tau}_{3}\right)$ there exists no Pareto-improving parental intervention, because in period 1 type $\dot{\tau}_{1}$ is mistakenly certain there that the state is $\left(\dot{\tau}_{1}, \ddot{\tau}_{2}, \ddot{\tau}_{3}\right)$ and expects the backward induction path there $\hat{x}_{\left(\dot{\tau}_{1}, \dot{\tau}_{2}, \ddot{z}_{3}\right)}$. However, if the parent attaches equal importance to the well-being of the child in all three periods, that is, has the same utility $v$ as in Example 1, there do exist $x \neq \hat{x}_{\left(\dot{\tau}_{1}, \ddot{\tau}_{2}, \ddot{z}_{3}\right)}$ with which $v(x)>v\left(\hat{x}_{\left(\dot{\tau}_{1}, \dot{\tau}_{2}, \dot{\tau}_{3}\right)}\right)$ (see the details in Appendix A). Nevertheless, as long as the parent confines herself to interventions that all selves would consider to be Pareto-improving given their (possibly misguided) beliefs, the parent's hands are tied.

This is not a knife-edge phenomenon. Rather, it is resilient to some perturbations of self 1's beliefs. For example, for $\varepsilon>0$ small enough (in fact, for $\varepsilon \leq \frac{1}{2}$ ), even if $\dot{\tau}_{1}$ ascribes probability $\varepsilon$ to the prevailing state of the world $\left(\dot{\tau}_{1}, \dot{\tau}_{2}, \dot{\tau}_{3}\right)$, that is, 


$$
\begin{array}{ll}
\beta_{\dot{\tau}_{1}}\left\{\left(\dot{\tau}_{1}, \dot{\tau}_{2}, \dot{\tau}_{3}\right)\right\}=\varepsilon, & \beta_{\dot{\tau}_{1}}\left\{\left(\dot{\tau}_{1}, \ddot{\tau}_{2}, \ddot{\tau}_{3}\right)\right\}=1-\varepsilon \\
\beta_{\dot{\tau}_{2}}\left\{\left(\dot{\tau}_{1}, \dot{\tau}_{2}, \dot{\tau}_{3}\right)\right\}=1, & \beta_{\ddot{\tau}_{2}}\left\{\left(\dot{\tau}_{1}, \ddot{\tau}_{2}, \ddot{\tau}_{3}\right)\right\}=1 \\
\beta_{\dot{\tau}_{3}}\left\{\left(\dot{\tau}_{1}, \dot{\tau}_{2}, \dot{\tau}_{3}\right)\right\}=1, & \beta_{\ddot{t}_{3}}\left\{\left(\dot{\tau}_{1}, \ddot{\tau}_{2}, \ddot{\tau}_{3}\right)\right\}=1
\end{array}
$$

there still do not exist parental interventions which would be Pareto-improving over backward induction. The details are elaborated in Appendix A.

\section{1 | Nudge}

Instead of influencing the choice path given the misperceptions of the child, the parent can try to intervene ex ante with information, by drawing the attention of the child at the state $\left(\dot{\tau}_{1}, \dot{\tau}_{2}, \dot{\tau}_{3}\right)$ to the actual utilities there $\left(u_{i_{1}}, u_{i_{2}}, u_{i_{3}}\right)$. That would amount to altering the belief $\beta_{i_{1}}$ of the type $\dot{\tau}_{1}$, by increasing the probability $\varepsilon$ that it ascribes to $\left(\dot{\tau}_{1}, \dot{\tau}_{2}, \dot{\tau}_{3}\right)$.

Such informational, noncoercive interventions are a particular form of a nudge (Thaler \& Sunstein, 2008). In case the nudge is fully successful, $\varepsilon=1$, the child becomes sophisticated and anticipates correctly his forthcoming preference change.

However, as $\varepsilon$ increases, $\dot{\tau}_{1}$ becomes more disillusioned, and as a result its expected backward-induction well-being $\hat{u}_{\dot{t}_{1}}$ decreases (see the details in Appendix A). Thus, even though the nudge does not interfere with the choice $x$ of the child by coercion or incentives, the sobering effect of the nudge causes a backlash to the well-being of the child in period 1, and for no $\varepsilon>0$ would the nudge induce a Pareto improvement.

\section{5 | MINIMALLY DISAPPOINTING INTERVENTIONS}

Situations as in Example 2 put the parent in a very frustrating position: due to the child's misperceptions about his future preferences, there is no way for the parent to ameliorate the aggregate well-being of the child without upsetting him at least in one period. This raises the question whether the Pareto-improvement constraint is not too stringent under such misperceptions.

A path $x$ which is Pareto-improving at a state of the world $\tau$ vis-à -vis backward induction satisfies, by definition, $u_{\tau_{i}}(x)-\hat{u}_{\tau_{i}} \geq 0$ for $i=1, \ldots, n$. A natural way to minimally relax this constraint is to require

$$
u_{\tau_{i}}(x)-\hat{u}_{\tau_{i}} \geq-\delta, \quad i=1, \ldots, n
$$

for the minimal $\delta$ with which a path $x$ satisfying $(\sharp)$ exists.

Then, if for this

$$
\delta_{\min }(\tau) \equiv \min \left\{\delta: \exists x \quad \text { s.t. } \quad u_{\tau_{i}}(x)-\hat{u}_{\tau_{i}} \geq-\delta, i=1, \ldots, n\right\}
$$

it is the case that

$$
\max _{\left\{x: \min _{i=1, \ldots, n}\left(u_{\tau_{i}}(x)-\hat{u}_{\tau_{i}}\right) \geq-\delta_{\min }(\tau)\right\}} v(x)>v\left(\hat{x}_{\tau}\right)
$$

then the parent may find it legitimate to intervene and induce 


$$
\tilde{x} \in \arg \max _{\left\{x: \min _{i=1, \ldots, n}\left(u_{\tau_{i}}(x)-\hat{u}_{\tau_{i}}\right) \geq-\delta_{\min }(\tau)\right\}} v(x)
$$

to augment the aggregate well-being of the child, even though at some period the child will be minimally disappointed, by $\delta_{\min }(\tau)$.

Example 2 (Continued). In the state of the world $\left(\dot{\tau}_{1}, \dot{\tau}_{2}, \dot{\tau}_{3}\right)$ there turns out to be a unique path $\tilde{x}$ satisfying

$$
u_{\dot{\tau}_{i}}(x)-\hat{u}_{\dot{\tau}_{i}} \geq-\delta_{\min }\left(\dot{\tau}_{1}, \dot{\tau}_{2}, \dot{\tau}_{3}\right) \quad i=1,2,3
$$

(see the details in Appendix A). With this path $\tilde{x}$,

$$
\begin{aligned}
& u_{\dot{\tau}_{1}}(\tilde{x})-\hat{u}_{\dot{\tau}_{1}}=-\delta_{\min }\left(\dot{\tau}_{1}, \dot{\tau}_{2}, \dot{\tau}_{3}\right) \\
& u_{\dot{\tau}_{2}}(\tilde{x})-\hat{u}_{\dot{\tau}_{2}}=-\delta_{\min }\left(\dot{\tau}_{1}, \dot{\tau}_{2}, \dot{\tau}_{3}\right)
\end{aligned}
$$

but

$$
u_{\dot{\tau}_{3}}(\tilde{x})-\hat{u}_{\dot{\tau}_{3}}>2 \delta_{\min }\left(\dot{\tau}_{1}, \dot{\tau}_{2}, \dot{\tau}_{3}\right)
$$

Thus,

$$
v(\tilde{x})>v\left(\hat{x}_{\left(\dot{\tau}_{1}, \dot{\tau}_{2}, \dot{\tau}_{3}\right)}\right),
$$

that is, in terms of average well-being across the three periods, which is the parent's perspective, the minimally disappointing intervention is superior to backward induction.

The approach proposed here may actually be applied also when Pareto improving interventions over backward induction $d o$ exist. With this approach, $\delta_{\min }$ would have the opposite sign and measure the minimal utility enhancement across the periods. The parent would then consider inducing only Pareto improving paths that maximally improve the minimal well-being enhancements across the different periods. In Appendix A we demonstrate how this idea is made operative in Example 1.

\section{6 | DISCUSSION}

\section{1 | Parent uncertainty}

We assumed, for simplicity, that the parent knows correctly the state of the world, that is, that the parent knows the child's preferences and beliefs in all periods. We saw that even under such a simplifying assumption, Pareto improving interventions might not be available to the parent. A fortiori, a parent who, more realistically, only has some belief about the states of the world, might have an even narrower scope for interventions which would be Pareto improving or minimally disappointing in all the states that she considers possible. How this scope narrows down with the parent's uncertainty remains open for follow-up inquiry.

\section{2 | State-contingent parental interventions}

We assumed, again for simplicity, that if the parent induces the child to follow a particular path $x$, this forthcoming path becomes known to the child, and that very fact might upset the child 
in some periods. Such upsetting could be potentially attenuated if the parent were allowed to make state-contingent empty promises, like

I know you are certain that tomorrow your present bias will disappear. I am certain that you are too optimistic about this, but if it so happens that I was wrong and you were right, then as of tomorrow I will alter the path so as to make it up for you as much as possible.

Notice, though, that comes tomorrow, it might be costly or even impossible to verify the time preference of the child, and in such case such a promise would be not only empty (under our assumption that the parent anticipates correctly the future preferences of the child), but also not credible in the first place.

\section{3 | Alternative normative guidelines}

When Pareto improving interventions are lacking, we proposed one possible normative constraint, namely minimizing the disappointment of the child across the time periods relative to the benchmark of its (expected) utility under the backward induction path, that he would follow absent of any parental intervention. An alternative normative constraint could be maximizing the minimal overall well-being across the time periods. Or one could even claim that in the absence of Pareto improving interventions, the parent should simply take the lead and induce a path that maximizes the parent's own view of how to balance the considerations of the child's well-being across the time periods, without imposing on herself any further normative constraints.

To conclude, we introduced a type-space framework for beliefs (and beliefs about beliefs, etc.) about one's future preferences, with the implied backward induction choice that each type will make given its belief. We proved that with sophistication, that is, when the state of the world is common knowledge, with all types agreeing with one another about future preferences and beliefs, then generically a parent has room for committing to induce paths which will Pareto-improve well-being across the time periods.

In contrast, with misperceptions about future preferences, such Pareto-improving interventions need not exist, and even informational nudges might be necessarily disillusioning at least in some time period. For such cases we proposed a possible normative alternative to the Pareto criterion, namely minimizing the maximal disappointment that the intervention entails across the time periods.

\section{ORCID}

Aviad Heifetz (D) http://orcid.org/0000-0003-0877-7263

\section{REFERENCES}

Arnold., V. I., Gusein-Zade, S. M., \& Varchenko, A. N. (1985). Singularities of differentiable maps (Vol. I). Birkhäuser.

Alós-Ferrer, C., \& Ritzberger, K. (2016). Equilibrium existence for large perfect information games. Journal of Mathematical Economics, 62, 5-18.

Dubey, P. (1986). Inefficiency of nash equilibria. Mathematics of Operations Research, 11(1), 1-8. 
Ericson, K. M., \& Laibson, D. (2019). Intertemporal choice. In B. Douglas Bernheim, Stefano DellaVigna, David Laibson Handbook of behavioral economics: Applications and foundations 1 (Vol. 2, pp. 1-67). North-Holland.

Goldman, S. M. (1979). Intertemporally inconsistent preferences and the rate of consumption. Econometrica, 47(3), 621-626.

Goldman, S. M. (1980). Consistent plans. The Review of Economic Studies, 47(3), 533-537.

Harris, C. (1985). Existence and characterization of perfect equilibrium in games of perfect information. Econometrica, 53(3), 613-628.

Hellwig, M., \& Leininger, W. (1987). On the existence of subgame-perfect equilibrium in infinite-action games of perfect information. Journal of Economic Theory, 43(1), 55-75.

Hellwig, M., Leininger, W., Reny, P. J., \& Robson, A. J. (1990). Subgame perfect equilibrium in continuous games of perfect information: An elementary approach to existence and approximation by discrete games. Journal of Economic Theory, 52(2), 406-422.

Hirsch, M. W. (1976). Differential topology, Volume 33 of Graduate texts in mathematics. Springer-Verlag.

Jackson, M. O., \& Yariv, L. (2014). Present bias and collective dynamic choice in the lab. American Economic Review, 104(12), 4184-4204.

Jackson, M. O., \& Yariv, L. (2015). Collective dynamic choice: The necessity of time inconsistency. American Economic Journal: Microeconomics, 7(4), 150-78.

Kang, M. (2019). Pareto-improving tax policies under hyperbolic discounting. International Tax and Public Finance, 26(3), 618-660.

Kang, M., \& Wang, L. (2019). Pareto criterion and long-term perspective criterion under myopic discounting. Economics Bulletin, 39(1), 24-32.

Kang, M., \& Ye, L. S. (2019). Present bias and corporate tax policies. Journal of Public Economic Theory, 21(2), 265-290.

Laibson, D. (1997). Golden eggs and hyperbolic discounting. The Quarterly Journal of Economics, 112(2), 443-478.

Mas-Colell, A. (1989). The theory of general economic equilibrium: A differentiable approach. Cambridge University Press.

Mertens, J. F., \& Rubinchik, A. (2017). Discounting and welfare evaluation of policies, Journal of Public Economic Theory, 19, 903-920.

Nagata, R. (2004). Theory of regular economies. World Scientific.

O'Donoghue, T., \& Rabin, M. (1999). Doing it now or later. American Economic Review, 89(1), $103-124$.

Phelps, E., \& Pollak, R. (1968). On second-best national saving and game-equilibrium growth. The Review of Economic Studies, 35(2), 185-199.

Pollak, R. A. (1968). Consistent planning. The Review of Economic Studies, 35(2), 201-208.

Strotz, R. H. (1955). Myopia and inconsistency in dynamic utility maximization. The Review of Economic Studies, 23(3), 165-180.

Strulik, H. (2021). Hyperbolic discounting and the time-consistent solution of three canonical environmental problems. Journal of Public Economic Theory, to appear, 23(3), 462-486. https://doi.org/10.1111/jpet.12497

Thaler, R. H., \& Sunstein, C. R. (2008). Nudge: Improving decisions about health, wealth, and happiness. Yale University Press.

How to cite this article: Heifetz, A., Minelli, E., \& Polemarchakis, H. (2021). Liberal parentalism. J Public Econ Theory. 23, 1107-1129. https://doi.org/10.1111/jpet.12517 


\section{APPENDIX A}

\section{A.1 | Proof of the Theorem}

For simplicity, we first present the proof for the case where the choice set of each self is onedimensional, $X_{i} \subset \mathbb{R}^{k_{i}}$ with $k_{i}=1, i=1, \ldots, n$, and then elaborate on how to read the same proof for the case of any finite $k_{i} \geq 1$.

If $\hat{x}=\left(\hat{x}_{1}, \ldots, \hat{x}_{n}\right)$ is an interior SPE path, then at $\hat{x}$ the following $n$ first-order conditions obtain:

$$
\begin{array}{r}
\frac{\partial u_{n}}{\partial x_{n}}=0 \\
\frac{\partial u_{n-1}}{\partial x_{n-1}}+\frac{\partial u_{n-1}}{\partial x_{n}} \frac{\partial b_{n}}{\partial x_{n-1}}=0 \\
\frac{\partial u_{n-2}}{\partial x_{n-2}}+\frac{\partial u_{n-2}}{\partial x_{n-1}} \frac{\partial b_{n-1}}{\partial x_{n-2}}+\frac{\partial u_{n-2}}{\partial x_{n}}\left(\frac{\partial b_{n}}{\partial x_{n-2}}+\frac{\partial b_{n}}{\partial x_{n-1}} \frac{\partial b_{n-1}}{\partial x_{n-2}}\right)=0
\end{array}
$$

where for $k<\ell$, the derivatives of the best reply functions $\frac{\partial b_{\ell}}{\partial x_{k}}$ at $\hat{x}$ involve second (cross) derivatives of $u_{1}, \ldots u_{n}$.

To simplify notation in the sequel, define the matrix of direct and indirect effects

$$
h=\left(\begin{array}{cccccc}
1 & & \cdots & & & \vdots \\
0 & 1 & & & & \\
\vdots & & \ddots & & & \\
0 & & 0 & 1 & \frac{\partial b_{n-1}}{\partial x_{n-2}} & \left(\frac{\partial b_{n}}{\partial x_{n-2}}+\frac{\partial b_{n}}{\partial x_{n-1}} \frac{\partial b_{n-1}}{\partial x_{n-2}}\right) \\
0 & \cdots & & 0 & 1 & \frac{\partial b_{n}}{\partial x_{n-1}} \\
0 & & \cdots & & 0 & 1
\end{array}\right)
$$

so that the above system becomes at $\hat{x}$

$$
\begin{aligned}
\frac{\partial u_{n}}{\partial x_{n}} & =0 \\
\frac{\partial u_{n-1}}{\partial x_{n-1}}+\frac{\partial u_{n-1}}{\partial x_{n}} h_{n-1, n} & =0 \\
\frac{\partial u_{n-2}}{\partial x_{n-2}}+\frac{\partial u_{n-2}}{\partial x_{n-1}} h_{n-2, n-1}+\frac{\partial u_{n-2}}{\partial x_{n}} h_{n-2, n} & =0
\end{aligned}
$$

If $\hat{x}$ is also Pareto optimal, then at $\hat{x}$ the rows of the Jacobian

$$
D u=\left(\begin{array}{c}
\nabla u_{1} \\
\vdots \\
\nabla u_{n}
\end{array}\right)=\left(\begin{array}{ccc}
\frac{\partial u_{1}}{\partial x_{1}} & \cdots & \frac{\partial u_{1}}{\partial x_{n}} \\
\vdots & \ddots & \vdots \\
\frac{\partial u_{1}}{\partial x_{n}} & \cdots & \frac{\partial u_{n}}{\partial x_{n}}
\end{array}\right)
$$


are linearly dependent-otherwise one can find a direction $\left(\begin{array}{c}\delta_{1} \\ \vdots \\ \delta_{n}\end{array}\right)$ such that $\left(\begin{array}{c}\nabla u_{1} \\ \vdots \\ \nabla u_{n}\end{array}\right)\left(\begin{array}{c}\delta_{1} \\ \vdots \\ \delta_{n}\end{array}\right)=\left(\begin{array}{c}1 \\ \vdots \\ 1\end{array}\right)$, so that perturbing $\hat{x}_{i}$ in the direction $\delta_{i}$ for $i=1, \ldots, n$ improves all of $u_{1}, \ldots u_{n}$, thus constituting a Pareto improvement. Hence, if $\hat{x}$ is Pareto optimal there exists $\hat{\theta}=\left(\hat{\theta}_{1}, \ldots, \hat{\theta}_{n}\right) \neq 0$ such $\hat{\theta} D u(\hat{x})=0$. The vector $\hat{\theta}$ therefore belongs to one of the two following families of cases:

(i) There exists a subset of indices $1 \leq \ell_{1}<\cdots<\ell_{k} \leq n$ such that $\hat{\theta}_{\ell_{1}} \neq 0, \ldots, \hat{\theta}_{\ell_{k}} \neq 0$.

(ii) There exists a unique index $1 \leq c \leq n$ such that $\hat{\theta}_{c} \neq 0$.

To account for the first family of cases, for each subset of indices $1 \leq \ell_{1}<\cdots<\ell_{k} \leq n$ consider the map

$$
\left(F_{1}, \ldots, F_{n}, G_{1}, \ldots G_{n}, G_{\left\{\ell_{1}, \ldots, \ell_{k}\right\}}\right): X \times \mathbb{R}^{n} \times \mathcal{U} \rightarrow \mathbb{R}^{2 n+1}
$$

defined by

$$
\begin{gathered}
F_{n}(x, \theta ; u) \equiv \frac{\partial u_{n}(x)}{\partial x_{n}} \\
F_{n-1}(x, \theta ; u) \equiv \frac{\partial u_{n-1}(x)}{\partial x_{n-1}}+\frac{\partial u_{n-1}(x)}{\partial x_{n}} h_{n-1, n}(x) \\
F_{n-2}(x, \theta ; u) \equiv \frac{\partial u_{n-2}(x)}{\partial x_{n-2}}+\frac{\partial u_{n-2}(x)}{\partial x_{n-1}} h_{n-2, n-1}(x)+\frac{\partial u_{n-2}(x)}{\partial x_{n}} h_{n-2, n}(x) \\
\vdots \\
G_{1}(x, \theta ; u) \equiv \sum_{i=1}^{n} \theta_{i} \frac{\partial u_{i}(x)}{\partial x_{1}} \\
\vdots \\
G_{n}(x, \theta ; u) \equiv \sum_{i=1}^{n} \theta_{i} \frac{\partial u_{i}(x)}{\partial x_{n}} \\
-\left(B I_{\mathrm{n}-1}\right) \\
G_{\left\{\ell_{1}, \ldots, \ell_{k}\right\}}(x, \theta ; u) \equiv \theta_{\ell_{1}}^{2} \ldots \theta_{\ell_{k}}^{2} \\
-1
\end{gathered}
$$

Since the best-reply functions $b_{i}$ and hence the indirect effects $h_{i k}$ involve up-to second order cross-derivatives of $u_{i}$, we can view the map $\left(F_{1}, \ldots, F_{n}, G_{1}, \ldots, G_{n}, G_{\left\{\ell_{1}, \ldots, \ell_{k}\right\}}\right)$ as defined ${ }^{19}$ on the image of the 2-jet extensions

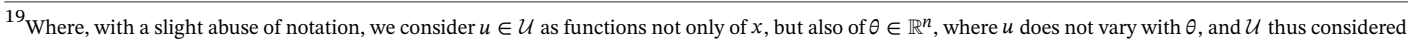
as a submanifold of $C^{\infty}\left(\mathbb{R}^{2 n}, \mathbb{R}^{n}\right)$.
} 


$$
\begin{aligned}
j_{(x, \theta)}^{2} u= & \left(x, \theta, u_{i}(x,), \frac{\partial u_{i}(x, \theta)}{\partial x_{k}}, \frac{\partial u_{i}(x, \theta)}{\partial \theta_{k}}, \frac{\partial u_{i}^{2}(x, \theta)}{\partial x_{k} \partial x_{\ell}}, \frac{\partial u_{i}^{2}(x, \theta)}{\partial x_{k} \partial \theta_{m}}, \frac{\partial u_{i}^{2}(x, \theta)}{\partial \theta_{k} \partial \theta_{\ell}}\right)_{\substack{i, k, \ell, m=1, \ldots, n \\
k \leq \ell}} \\
& \in J^{2}\left(\mathbb{R}^{2 n}, \mathbb{R}^{n}\right) .
\end{aligned}
$$

We will show below that $0 \in \mathbb{R}^{2 n+1}$ is a regular value of the $\operatorname{map}\left(F_{1}, \ldots, F_{n}, G_{1}, \ldots, G_{n}, G_{\left\{\ell_{1}, \ldots, \ell_{k}\right\}}\right)$. By the preimage theorem (see e.g., Nagata, 2004, theorem 2.1), that $0 \in \mathbb{R}^{2 n+1}$ is a regular value of $\left(F_{1}, \ldots, F_{n}, G_{1}, \ldots, G_{n}, G_{\left\{\ell_{1}, \ldots, \ell_{k}\right\}}\right)$ implies that $\left(F_{1}, \ldots, F_{n}, G_{1}, \ldots, G_{n}, G_{\left\{\ell_{1}, \ldots, \ell_{k}\right\}}\right)^{-1}(0)$ is a closed submanifold of $J^{2}\left(\mathbb{R}^{2 n}, \mathbb{R}^{n}\right)$. Therefore, by the strong (Thom) transversality theorem (see e.g.,

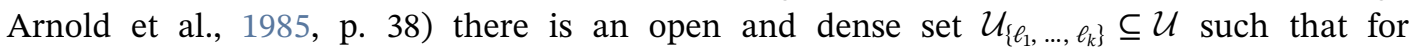
every $u \in \mathcal{U}_{\left\{\ell_{1}, \ldots, \ell_{k}\right\}}$,

$$
j^{2} u: \mathbb{R}^{2 n} \rightarrow J^{2}\left(\mathbb{R}^{2 n}, \mathbb{R}^{n}\right)
$$

that maps $(x, \theta) \mapsto j_{(x, \theta)}^{2} u$ is transversal to the submanifold $\left(F_{1}, \ldots, F_{n}, G_{\ell_{1}}, \ldots G_{\ell_{k}}, G_{\left\{\ell_{1}, \ldots, \ell_{k}\right\}}\right)^{-1}(0)$, and hence $0 \in \mathbb{R}^{2 n+1}$ is a regular value of the composed map

$$
\left(F_{1}, \ldots, F_{n}, G_{1}, \ldots, G_{n}, G_{\left\{\ell_{1}, \ldots, \ell_{k}\right\}}\right) \circ j^{2} u: \mathbb{R}^{2 n} \rightarrow \mathbb{R}^{2 n+1} .
$$

Since $\operatorname{dim}\left(\mathbb{R}^{2 n+1}\right)>\operatorname{dim}\left(\mathbb{R}^{2 n}\right)$, that $0 \in \mathbb{R}^{2 n+1}$ is a regular value then means that for every $u \in \mathcal{U}_{\left\{\ell_{1}, \ldots, \ell_{k}\right\}}$,

$$
\left(F_{1}, \ldots, F_{n}, G_{1}, \ldots, G_{n}, G_{\left\{\ell_{1}, \ldots, \ell_{k}\right\}}\right)(\hat{x}, \hat{\theta} ; u) \neq 0
$$

for every $(\hat{x}, \hat{\theta}) \in \mathbb{R}^{2 n}$.

To account for the second family of cases, for every $1 \leq c \leq n$ consider the map

$$
\left(F_{1}, \ldots, F_{c-1}, F_{c+1}, \ldots F_{n}, G_{1}, \ldots, G_{n}, G_{\{c\}}\right): \mathbb{R}^{n+1} \times \mathcal{U} \rightarrow \mathbb{R}^{2 n},
$$

where $F_{c}$ does not appear (because when $c$ is the unique index for which $\hat{\theta}_{c} \neq 0$ in the vector $\hat{\theta}$ satisfying $\hat{\theta} D u(\hat{x})=0$, notice that $\hat{\theta}_{c} \nabla u_{c}(\hat{x})=0$, and hence a fortiori $F_{c}(\hat{x}, \hat{\theta} ; u)=0$ as well), and $\theta_{\ell} \equiv 0$ for $\ell \neq c$. We will show below that $0 \in \mathbb{R}^{2 n}$ is a regular value of the map $\left(F_{1}, \ldots, F_{c-1}, F_{c+1}, \ldots F_{n}, G_{1}, \ldots, G_{n}, G_{\{c\}}\right)$, implying that there is an open and dense subset $\mathcal{U}_{\{c\}} \subseteq \mathcal{U}$ such that for every $u \in \mathcal{U}_{\{c\}}, 0 \in \mathbb{R}^{2 n}$ is a regular value of

$$
\left(F_{1}, \ldots, F_{c-1}, F_{c+1}, \ldots F_{n}, G_{1}, \ldots, G_{n}, G_{\{c\}}\right) \circ j^{2} u: \mathbb{R}^{n+1} \rightarrow \mathbb{R}^{2 n} .
$$

Since $\operatorname{dim}\left(\mathbb{R}^{2 n}\right)>\operatorname{dim}\left(\mathbb{R}^{n+1}\right)$, that $0 \in \mathbb{R}^{2 n}$ is a regular value means that for every $u \in \mathcal{U}_{\{c\}}$,

$$
\left(F_{1}, \ldots, F_{c-1}, F_{c+1}, \ldots F_{n}, G_{1}, \ldots, G_{n}, G_{\{c\}}\right)\left(\hat{x}, \hat{\theta}_{c} ; u\right) \neq 0
$$

for every $\left(\hat{x}, \hat{\theta}_{c}\right) \in \mathbb{R}^{n+1}$.

The intersection

$$
\mathcal{U}_{0}=\bigcap_{S \in 2^{\{1, \ldots, n\}} \backslash \varnothing} \mathcal{U}_{S}
$$

is thus open and dense, and for every $u \in \mathcal{U}_{0}$ and every interior SPE $\hat{x}$ (satisfying $F_{1}(\hat{x}, \cdot ; u)=\cdots=F_{n}(\hat{x}, \cdot ; u)=0$ ) there exists no $\hat{\theta} \neq 0$ for which $\hat{\theta} D u(\hat{x})=0$ (i.e., satisfying, for the set of indices $S$ of $\hat{\theta}$ 's nonzero entries, $\left.G_{1}(\hat{x}, \hat{\theta} ; u)=\cdots=G_{n}(\hat{x}, \hat{\theta} ; u)=G_{S}(\hat{x}, \hat{\theta} ; u)=0\right)$. That is, for every $u \in \mathcal{U}_{0}$, no interior SPE $\hat{x}$ is Pareto optimal, as required.

In the first family of cases, to prove that $0 \in \mathbb{R}^{2 n+1}$ is indeed a regular value of the map $\left(F_{1}, \ldots, F_{n}, G_{1}, \ldots G_{n}, G_{\left\{\ell_{1}, \ldots, \ell_{k}\right\}}\right)$, we will show that for every $j_{(\hat{x}, \hat{\theta})}^{2} \hat{u} \in\left(F_{1}, \ldots, F_{n}, G_{1}, \ldots G_{\left\{\ell_{1}, \ldots, \ell_{k}\right\}}\right)^{-1}(0)$ and for every perturbation direction $\left(\pi_{1}, \ldots, \pi_{n}, p_{1}, \ldots, p_{n}, p_{n+1}\right)$ of $0 \in \mathbb{R}^{2 n+1}$ there exist 


$$
Y=\left(\begin{array}{ccc}
y_{11} & \cdots & y_{1 n} \\
\vdots & & \vdots \\
y_{n 1} & \cdots & y_{n n}
\end{array}\right) \in \mathbb{R}^{n^{2}}, \quad z=\left(\begin{array}{c}
z_{1} \\
\vdots \\
z_{n}
\end{array}\right) \in \mathbb{R}^{n}
$$

such that with the paths of utility profiles and parameter values

$$
u^{t}=\left(\begin{array}{c}
u_{1}^{t} \\
\vdots \\
u_{n}^{t}
\end{array}\right) \equiv \hat{u}+t Y\left(\begin{array}{c}
x_{1} \\
\vdots \\
x_{n}
\end{array}\right), \quad \theta^{t}=\left(\begin{array}{c}
\theta_{1}^{t} \\
\vdots \\
\theta_{n}^{t}
\end{array}\right) \equiv \hat{\theta}+t\left(\begin{array}{c}
z_{1} \\
\vdots \\
z_{n}
\end{array}\right)
$$

defined for $t \in[-\varepsilon, \varepsilon]$ for some $\varepsilon>0$, at $t=0$ we have

$$
\begin{aligned}
& {\frac{\partial F_{1}\left(j_{\left(\hat{x}, \theta^{t}\right)}^{2} u^{t}\right)}{\partial t} \quad \mid t=0}=\pi_{1} \\
& {\frac{\partial F_{n}\left(j_{\left(\hat{x}, \theta^{t}\right)}^{2} u^{t}\right)}{\partial t}}_{\mid t=0}=\pi_{n} \\
& \frac{\partial G_{1}\left(j_{\left(\hat{x}, \theta^{t}\right)}^{2} u^{t}\right)}{\partial t \quad}=p_{1} \\
& \frac{\partial G_{n}\left(j_{\left(\hat{x}, \theta^{t}\right)}^{2} u^{t}\right)}{\partial t \quad}=p_{n} \\
& \frac{\partial G_{\left\{\ell_{1}, \ldots, \ell_{k}\right\}}\left(j_{\left(\hat{x}, \theta^{t}\right)}^{2} u^{t}\right)}{\partial t}{ }_{\mid t=0}=p_{n+1}
\end{aligned}
$$

To this effect, denote $a=\ell_{1}, b=\ell_{k}$ and choose $z$ to be

$$
z=\left(\begin{array}{c}
0 \\
\vdots \\
z_{b} \\
\vdots \\
0
\end{array}\right)
$$

with $z_{b}=\frac{p_{n+1}}{2 \hat{\theta}_{b} \hat{\theta}_{\ell_{1}}^{2} \cdots \hat{\theta}_{\ell_{k-1}}^{2}}$, and $Y$ to be

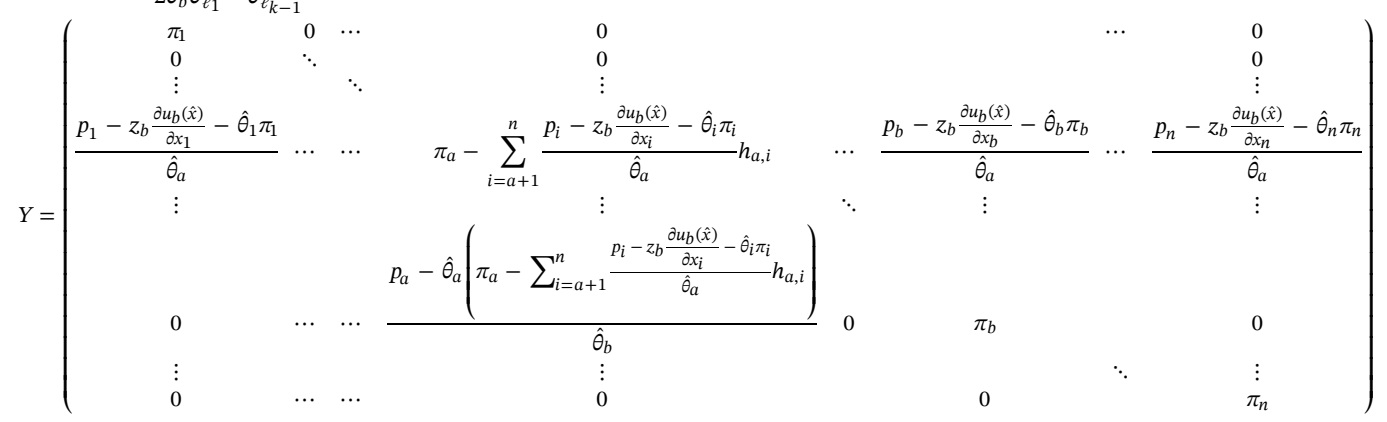


In words,

(0) Set $z_{b}=\frac{p_{n+1}}{2 \hat{\theta}_{b} \hat{\theta}_{\ell_{1}}^{2} \cdots \hat{\theta}_{\ell_{k-1}}^{2}}$, thus perturbing $G_{\left\{\ell_{1}, \ldots, \ell_{k}\right\}}$ at $t=0$ by $p_{n+1}$.

(1) For $i \neq a$ set $y_{i i}=\pi_{i}$, thus perturbing $F_{i}$ at $t=0$ by $\pi_{i}$ for $i \neq a$;
(2) For $i \neq a$, (0) and (1) perturb $G_{i}$ at $t=0$ by $z_{b} \frac{\partial u_{b}(\hat{x})}{\partial x_{i}}+\hat{\theta}_{i} \pi_{i}$, so set $y_{a i}=\frac{p_{i}-z_{b} \frac{\partial u_{b}(\hat{x})}{\partial x_{i}}-\hat{\theta}_{i} \pi_{i}}{\hat{\theta}_{a}}$ to

eventually end up perturbing $G_{i}$ at $t=0$ by $p_{i}$;
(3) Together over all $i \neq a$, (2) upsets $F_{a}$ at $t=0$ by $\sum_{i>a} \frac{p_{i}-z_{b} \frac{\partial u_{b}(\hat{x})}{\partial x_{i}}-\hat{\theta}_{i} \pi_{i}}{\hat{\theta}_{a}} h_{a, i}$ (only, $h_{a, i}$ itself was not perturbed, because it depends on partial cross-derivatives of $u_{r}$ w.r.t. $x_{s}$ for $r>s>a$, and these were not perturbed); so to eventually perturb $F_{a}$ at $t=0$ by $\pi_{a}$, set $y_{a a}=\pi_{a}-\sum_{i>a} \frac{p_{i}-z_{b} \frac{\partial u_{b}(\hat{x})}{\partial x_{i}}-\hat{\theta}_{i} \pi_{i}}{\hat{\theta}_{a}} h_{a, i}$;

(4) Finally, (3) upsets $G_{a}$ at $t=0$ by $\hat{\theta}_{a}\left(\pi_{a}-\sum_{i>a} \frac{p_{i}-z_{b} \frac{\partial u_{b}(\hat{x})}{\partial x_{i}}-\hat{\theta}_{i} \pi_{i}}{\hat{\theta}_{a}} h_{a, i}\right)$, so to eventually perturb $G_{a}$
at $t=0$ by $p_{a}$, set

$$
y_{b a}=\frac{p_{a}-\hat{\theta}_{a}\left(\pi_{a}-\sum_{i>a} \frac{p_{i}-z_{b} \frac{\partial u_{b}(\hat{x})}{\partial x_{i}}-\hat{\theta}_{i} \pi_{i}}{\hat{\theta}_{a}} h_{a, i}\right)}{\hat{\theta}_{b}}
$$

(This does not upset $F_{b}$, which only depends on partial derivatives of $u_{i b}$ w.r.t. $x_{s}$ for $s \geq b$, whereas $a<b$.)

(5) All the remaining entries of $Y$ are zero.

As for cases of the second family, to prove that $0 \in \mathbb{R}^{2 n}$ is a regular value of $\left(F_{1}, \ldots, F_{c-1}, F_{c+1}, \ldots F_{n}, G_{1}, \ldots, G_{n}, G_{\{c\}}\right)$, for every corresponding rates of perturbation $\left(\left(\pi_{k}\right)_{k \neq c},\left(p_{i}\right)_{i=1}^{n+1}\right) \in \mathbb{R}^{2 n}$, choose $z_{c}=\frac{p_{n+1}}{2 \hat{\theta}_{c}}$ so as to perturb $G_{\{c\}}$ at $t=0$ by $p_{n+1}$, and

$$
Y=\left(\begin{array}{ccccccc}
\pi_{1} & \cdots & 0 & \cdots & 0 & \cdots & 0 \\
\vdots & \ddots & \vdots & & \vdots & & \vdots \\
0 & \cdots & \pi_{j} & \cdots & 0 & \cdots & 0 \\
\vdots & & \vdots & \ddots & \vdots & & \vdots \\
\frac{p_{1}-z_{c} \frac{\partial u_{c}(\hat{x})}{\partial x_{1}}}{\hat{\theta}_{c}} & \cdots & \frac{p_{j}-z_{c} \frac{\partial u_{c}(\hat{x})}{\partial x_{j}}}{\hat{\theta}_{c}} & \cdots & \frac{p_{c}-z_{c} \frac{\partial u_{c}(\hat{x})}{\partial x_{c}}}{\hat{\theta}_{c}} & \frac{p_{n}-z_{c} \frac{\partial u_{c}(\hat{x})}{\partial x_{n}}}{\hat{\theta}_{c}} \\
\vdots & & \vdots & & \vdots & \ddots & \vdots \\
0 & \cdots & 0 & \cdots & 0 & \cdots & \pi_{n}
\end{array}\right)
$$

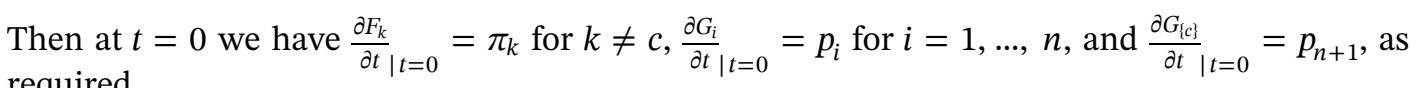
required.

When choice variables are multi-dimensional, $x_{i}=\left(x_{i, 1}, \ldots, x_{i, k_{i}}\right), i=1, \ldots, n$, the same proof applies verbatim with the following caveats:

$\frac{\partial u_{i}}{\partial x_{j}}=\left(\frac{\partial u_{i}}{\partial x_{j, 1}}, \ldots \frac{\partial u_{i}}{\partial x_{j, k_{j}}}\right), \frac{\partial b_{i}}{\partial x_{j}}=\left(\begin{array}{ccc}\frac{\partial b_{i, 1}}{\partial x_{j, 1}} & \cdots & \frac{\partial b_{i, 1}}{\partial x_{j, k_{j}}} \\ \vdots & & \vdots \\ \frac{\partial b_{i, k_{i}}}{\partial x_{j, 1}} & \cdots & \frac{\partial b_{i, k_{i}}}{\partial x_{j, k_{j}}}\end{array}\right), F_{i}=\left(F_{i, 1}, \ldots, F_{i, k_{i}}\right), \frac{\partial F_{i}}{\partial x_{j}}=\left(\begin{array}{ccc}\frac{\partial F_{i, 1}}{\partial x_{j, 1}} & \cdots & \frac{\partial F_{i, 1}}{\partial x_{j, k_{j}}} \\ \vdots & \vdots \\ \frac{\partial F_{i, k_{i}}}{\partial x_{j, 1}} & \cdots & \frac{\partial F_{i, k_{i}}}{\partial x_{j, k_{j}}}\end{array}\right)$, 
$\pi_{i}=\left(\pi_{i, 1}, \ldots, \pi_{i, k_{i}}\right), G_{i}=\left(G_{i, 1}, \ldots, G_{i, k_{i}}\right), \frac{\partial G_{i}}{\partial x_{j}}=\left(\begin{array}{ccc}\frac{\partial G_{i, 1}}{\partial x_{j, 1}} & \cdots & \frac{\partial G_{i, 1}}{\partial x_{j, k_{j}}} \\ \vdots & & \vdots \\ \frac{\partial G_{i, k_{i}}}{\partial x_{j, 1}} & \cdots & \frac{\partial G_{i, k_{i}}}{\partial x_{j, k_{j}}}\end{array}\right), p_{i}=\left(p_{i, 1}, \ldots, p_{i, k_{i}}\right)$,

$\delta_{i}=\left(\delta_{i, 1}, \ldots, \delta_{i, k_{i}}\right) ; \quad \theta_{i}$ remains a scalar. $\mathbb{R}^{2 n}$ is replaced by $\mathbb{R}^{k_{1}+\cdots+k_{n}+n}$, and $\mathbb{R}^{2 n+1}$ by $\mathbb{R}^{k_{1}+\cdots+k_{n}+n+1}$.

Remark 2. Our theorem is phrased in terms of generic utility profiles, but it holds also for generic preference profiles: The projection $\Phi$ from utility functions to the preference relations that they represent is a continuous, open map (Mas-Colell, 1989, p. 70, definition 2.4.1 and proposition 2.4.2), and therefore it maps $\mathcal{U}_{0}$ to an open and dense set of preference profiles with which no interior equilibrium is Pareto optimal.

\section{A.2 | Example 1 analysis}

\section{A.2.1 | Backward induction}

Self 3 has no effective choice but to complete what its preceding selves haven't done, $b_{3}\left(x_{1}, x_{2}\right)=3-x_{1}-x_{2}$. By backward induction, self 2 solves

$$
\max _{x_{2}} u_{2}\left(x_{1}, x_{2}, b_{3}\left(x_{1}, x_{2}\right)\right)=\max _{x_{2}}\left(\left(24-x_{1}^{2}\right)+\left(24-x_{2}^{2}\right)+\frac{1}{2}\left(24-\left(3-x_{1}-x_{2}\right)^{2}\right)\right)
$$

leading to

$$
b_{2}\left(x_{1}\right)=1-\frac{1}{3} x_{1}
$$

Accordingly, self 1 solves

$$
\begin{aligned}
& \max _{x_{1}} u_{1}\left(x_{1}, b_{2}\left(x_{1}\right), b_{3}\left(x_{1}, b_{2}\left(x_{1}\right)\right)\right) \\
= & \max _{x_{1}}\left(\left(24-x_{1}^{2}\right)+\frac{1}{2}\left(24-\left(1-\frac{1}{3} x_{1}\right)^{2}\right)+\frac{1}{2}\left(24-\left(3-x_{1}-\left(1-\frac{1}{3} x_{1}\right)\right)^{2}\right)\right)
\end{aligned}
$$

leading it to choose

$$
\hat{x}_{1}=\frac{15}{23}=0.6522 \text {. }
$$

Consequently,

$$
\hat{x}_{2}=b_{2}\left(\hat{x}_{1}\right)=\frac{18}{23}=0.7826
$$

and

$$
\hat{x}_{3}=b_{3}\left(\hat{x}_{1}, \hat{x}_{2}\right)=\frac{36}{23}=1.5652 \text {. }
$$

Altogether, the backward induction path is $\hat{x}=\left(\frac{15}{23}, \frac{18}{23}, \frac{36}{23}\right)$. The corresponding utilities are 


$$
\begin{aligned}
& \hat{u}_{1}=u_{1}(\hat{x})=46.044 \\
& \hat{u}_{2}=u_{2}(\hat{x})=57.737 \\
& \hat{u}_{3}=u_{3}(\hat{x})=68.512
\end{aligned}
$$

whose average is

$$
V(\hat{u})=v(\hat{x})=57.431
$$

\section{A.2.2 I The parent's problem}

With no normative constraints on interventions, the parent would maximize the average utility of the three selves

$$
v\left(x_{1}, x_{2}, x_{3}\right)=\frac{1}{3} \sum_{i=1}^{3} u_{i}\left(x_{1}, x_{2}, x_{3}\right)=\left(24-x_{1}^{2}\right)+\frac{5}{6}\left(24-x_{2}^{2}\right)+\frac{2}{3}\left(24-x_{3}^{2}\right)
$$

subject to the physical constraint $x_{1}+x_{2}+x_{3}=3$. This yields

$$
\bar{x} \equiv\left(\bar{x}_{1}, \bar{x}_{2}, \bar{x}_{3}\right)=\left(\frac{30}{37}, \frac{36}{37}, \frac{45}{37}\right)=(0.811,0.973,1.216) .
$$

The utility levels are

$$
\begin{aligned}
& u_{1}(\bar{x})=46.13 \\
& u_{2}(\bar{x})=57.656 \\
& u_{3}(\bar{x})=68.917
\end{aligned}
$$

whose average

$$
v(\bar{x})=57.568
$$

is higher than without intervention,

However, comparing $\left({ }^{* *}\right)$ to $(*)$ reveals that $\bar{x}$ is not a Pareto improvement relative to the backward induction path without intervention $\hat{x}$, because $u_{2}(\bar{x})<\hat{u}_{2}$.

\section{A.2.3 | Pareto-improving interventions}

If the parent restricts herself to interventions that are Pareto-improving relative the backward induction path $\hat{x}$, the parent solves

$$
\max _{x_{1}+x_{2}+x_{3}=3} v\left(x_{1}, x_{2}, x_{3}\right) \quad \text { s.t. } \quad u_{i}\left(x_{1}, x_{2}, x_{3}\right) \geq \hat{u}_{i}, \quad i=1,2,3
$$

yielding the unique solution

$$
\breve{x} \equiv\left(\breve{x}_{1}, \breve{x}_{2}, \breve{x}_{3}\right)=(0.780,0.828,1.392)
$$

accruing the utility levels 


$$
\begin{aligned}
& u_{1}(\breve{x})=46.080 \\
& u_{2}(\breve{x})=57.737 \\
& u_{3}(\breve{x})=68.768
\end{aligned}
$$

with the lower average

$$
v(\breve{x})=57.528<v(\bar{x})
$$

In particular, along the choice path $\breve{x}$ self 1 does more of the chore relative to the backward induction path $\hat{x}$, but (comparing $\left(^{*}\right)$ with $(* * *)$ ) self 1 is more than happy to do so knowing that self 2 will also work harder; self 2 is just indifferent working harder given that self 1 works harder; and self 3 is happier that less of the largest share of the chore is left for it to complete.

\section{A.2.4 | Maximin intervention}

If the parent restricts herself further, to an intervention that maximizes the minimal utility enhancement across the three selves relative to the no-intervention backward induction path $\hat{x}$,

$$
\max _{x_{1}+x_{2}+x_{3}=1} \min _{i=1,2,3}\left[u_{i}\left(x_{1}, x_{2}, x_{3}\right)-\hat{u}_{i}\right] .
$$

The maximin is attained at

$$
\tilde{x} \equiv\left(\tilde{x}_{1}, \tilde{x}_{2}, \tilde{x}_{3}\right)=(0.739,0.784,1.477)
$$

with utility levels

$$
\begin{array}{r}
u_{1}(\tilde{x})=46.056 \\
u_{\tau_{2}}^{\hat{1}}(\tilde{x})=57.748 \\
u_{\tau_{3}}^{\hat{1}}(\tilde{x})=68.657
\end{array}
$$

accruing a utility increment of 0.0115 to both self 1 and self 2 relative to the no-intervention backward induction path, even if with an average utility across the selves

$$
v(\tilde{x})=57.487
$$

which is lower than (3os). Nevertheless, comparing with $(\boldsymbol{\beta})$ the parent realizes that $v(\tilde{x})>v(\hat{x})$, so she judges the maximin intervention as preferable to no intervention at all.

\section{A.3 | Example 2 analysis}

\section{A.3.1 | Backward induction}

Both $\dot{\tau}_{3}$ and $\ddot{\tau}_{3}$ have no choice but to complete the part of the chore that the previous selves haven't completed,

$$
b_{\dot{t}_{3}}\left(x_{1}, x_{2}\right)=b_{\tau_{3}}\left(x_{1}, x_{2}\right)=3-x_{1}-x_{2} .
$$

As for self 2 , type $\ddot{\tau}_{2}$, who has no present bias and the same utility function $u_{3}$ as that of $\ddot{\tau}_{3}$, will divide the remaining chore equally among them, 


$$
b_{\ddot{t}_{2}}\left(x_{1}\right)=\frac{3-x_{1}}{2}
$$

whereas type $\dot{\tau}_{2}$, who does have a present bias with the utility function $u_{2}$ will, like self 2 in example 1 , choose only

$$
b_{\dot{t}_{2}}\left(x_{1}\right)=1-\frac{1}{3} x_{1}
$$

The naive type $\dot{\tau}_{1}$, who is certain that the subsequent types are $\ddot{\tau}_{2}, \ddot{\tau}_{3}$ will solve

$$
\begin{aligned}
\max _{x_{1}} u_{1}\left(x_{1}, b_{t_{2}}\left(x_{1}\right), b_{t_{3}}\left(x_{1}, b_{\tau_{2}}\left(x_{1}\right)\right)\right)= & \max _{x_{1}}\left(\left(24-x_{1}^{2}\right)+\frac{1}{2}\left(24-\left(\frac{3-x_{1}}{2}\right)^{2}\right)\right. \\
& \left.+\frac{1}{2}\left(24-\left(\frac{3-x_{1}}{2}\right)^{2}\right)\right)
\end{aligned}
$$

leading him to choose

$$
b_{\dot{\tau}_{1}}(\varnothing)=\hat{x}_{\dot{\tau}_{1}}=\frac{3}{5}
$$

believing that $\ddot{\tau}_{2}$ and $\ddot{\tau}_{3}$ will divide the remaining chore equally, each choosing $\frac{6}{5}$. However, at the state $\left(\dot{\tau}_{1}, \dot{\tau}_{2}, \dot{\tau}_{3}\right)$, type $\dot{\tau}_{2}$ will actually choose

$$
\hat{x}_{i_{2}}=b_{\dot{\tau}_{2}}\left(\hat{x}_{\dot{t}_{1}}\right)=1-\frac{1}{3} \cdot \frac{3}{5}=\frac{4}{5}
$$

leaving

$$
\hat{x}_{\dot{t}_{3}}=\frac{8}{5}
$$

of the chore to $\dot{\tau}_{3}$. At $\left(\dot{\tau}_{1}, \dot{\tau}_{2}, \dot{\tau}_{3}\right)$ the (expected) utilities will therefore be

$$
\begin{aligned}
& \hat{u}_{\dot{\tau}_{1}}=u_{i_{1}}\left(\frac{3}{5}, \frac{6}{5}, \frac{6}{5}\right)=46.2 \\
& \hat{u}_{\dot{\tau}_{2}}=u_{\dot{\tau}_{2}}\left(\frac{3}{5}, \frac{4}{5}, \frac{8}{5}\right)=57.72 \\
& \hat{u}_{\dot{\tau}_{3}}=u_{\dot{\tau}_{3}}\left(\frac{3}{5}, \frac{4}{5}, \frac{8}{5}\right)=68.44
\end{aligned}
$$

Thus, with no intervention by the parent, the resulting average utility at $\left(\dot{\tau}_{1}, \dot{\tau}_{2}, \dot{\tau}_{3}\right)$ will then be

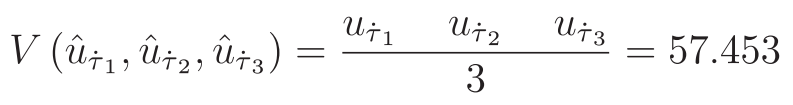

\section{A.3.2 | There exists no Pareto improving intervention}

At state $\left(\dot{\tau}_{1}, \dot{\tau}_{2}, \dot{\tau}_{3}\right)$ no intervention can be Pareto improving over the backward induction utility profile $\left(\hat{u}_{\dot{\tau}_{1}}, \hat{u}_{i_{2}}, \hat{u}_{\dot{\tau}_{3}}\right)$, because $\dot{\tau}_{1}$ believes that the state is $\left(\dot{\tau}_{1}, \ddot{\tau}_{2}, \ddot{\tau}_{3}\right)$ in which there is (common knowledge of) time consistency of preferences, and therefore by the principle of optimality in 
dynamic programming any alteration of the action profile $\left(\frac{3}{5}, \frac{6}{5}, \frac{6}{5}\right)$ chosen sequentially by $\dot{\tau}_{1}, \ddot{\tau}_{2}, \ddot{\tau}_{3}$ would be in particular unbeneficial from the perspective of $\dot{\tau}_{1}$. Moreover, since $u_{1}$ is strictly concave, any such alteration would actually decrease $u_{1}$.

The absence of Pareto improvements does not hinge on the fact that $\dot{\tau}_{1}$ is (wrongly) certain of (common knowledge of) the selves' time consistency. To see this, amend the above example so that type $\dot{\tau}_{1}$ does assign probability $\varepsilon>0$ that there is time inconsistency, thus:

$$
\begin{array}{ll}
\beta_{\dot{\tau}_{1}}\left\{\left(\dot{\tau}_{1}, \dot{\tau}_{2}, \dot{\tau}_{3}\right)\right\}=\varepsilon, & \beta_{\dot{\tau}_{1}}\left\{\left(\dot{\tau}_{1}, \ddot{\tau}_{2}, \ddot{\tau}_{3}\right)\right\}=1-\varepsilon \\
\beta_{\dot{\tau}_{2}}\left\{\left(\dot{\tau}_{1}, \dot{\tau}_{2}, \dot{\tau}_{3}\right)\right\}=1, & \beta_{\ddot{\tau}_{2}}\left\{\left(\dot{\tau}_{1}, \ddot{\tau}_{2}, \ddot{\tau}_{3}\right)\right\}=1 \\
\beta_{\dot{\tau}_{3}}\left\{\left(\dot{\tau}_{1}, \dot{\tau}_{2}, \dot{\tau}_{3}\right)\right\}=1, & \beta_{\ddot{\tau}_{3}}\left\{\left(\dot{\tau}_{1}, \ddot{\tau}_{2}, \ddot{\tau}_{3}\right)\right\}=1
\end{array}
$$

The maximization problem of $\dot{\tau}_{1}$ will now be

$$
\begin{aligned}
& \max _{x_{1}}\left(\varepsilon u_{\dot{t}_{1}}\left(x_{1}, b_{\dot{t}_{2}}\left(x_{1}\right), b_{\ddot{t}_{3}}\left(x_{1}, b_{\dot{\tau}_{2}}\left(x_{1}\right)\right)\right)+(1-\varepsilon) u_{\dot{\tau}_{1}}\left(x_{1}, b_{\ddot{t}_{2}}\left(x_{1}\right), b_{\ddot{t}_{3}}\left(x_{1}, b_{\ddot{t}_{2}}\left(x_{1}\right)\right)\right)\right) \\
& =\max _{x_{1}}\left(\begin{array}{l}
\varepsilon\left(\left(24-x_{1}^{2}\right)+\frac{1}{2}\left(24-\left(1-\frac{1}{3} x_{1}\right)^{2}\right)\right. \\
\left.+\frac{1}{2}\left(24-\left(3-x_{1}-\left(1-\frac{1}{3} x_{1}\right)\right)^{2}\right)\right) \\
+(1-\varepsilon)\left(\left(24-x_{1}^{2}\right)+\frac{1}{2}\left(24-\left(\frac{3-x_{1}}{2}\right)^{2}\right)+\frac{1}{2}\left(24-\left(\frac{3-x_{1}}{2}\right)^{2}\right)\right)
\end{array}\right)
\end{aligned}
$$

leading $\dot{\tau}_{1}$ to choose

$$
\hat{x}_{i_{1}}=\frac{3 \varepsilon+27}{\varepsilon+45}
$$

and subsequently

$$
\begin{aligned}
& \hat{x}_{\dot{t}_{2}}=b_{\dot{t}_{2}}\left(\hat{x}_{i_{1}}\right)=1-\frac{1}{3} \frac{3 \varepsilon+27}{\varepsilon+45}=\frac{36}{\varepsilon+45} \\
& \hat{x}_{\ddot{t}_{2}}=b_{\ddot{t}_{2}}\left(\hat{x}_{\dot{t}_{1}}\right)=\frac{3-\frac{3 \varepsilon+27}{\varepsilon+45}}{2}=\frac{54}{\varepsilon+45}
\end{aligned}
$$

leaving to the last self

$$
\begin{aligned}
& \hat{x}_{\dot{t}_{3}}=\frac{72}{\varepsilon+45} \\
& \hat{x}_{\ddot{t}_{3}}=\frac{54}{\varepsilon+45}
\end{aligned}
$$

Accordingly, the backward induction expected utilities at $\left(\dot{\tau}_{1}, \dot{\tau}_{2}, \dot{\tau}_{3}\right)$ will be

$$
\begin{aligned}
& \hat{u}_{\dot{\tau}_{1}}=\varepsilon u_{\dot{\tau}_{1}}\left(\frac{3 \varepsilon+27}{\varepsilon+45}, \frac{36}{\varepsilon+45}, \frac{72}{\varepsilon+45}\right)+(1-\varepsilon) u_{i_{1}}\left(\frac{3 \varepsilon+27}{\varepsilon+45}, \frac{54}{\varepsilon+45}, \frac{54}{\varepsilon+45}\right)=\frac{39 \varepsilon+2079}{\varepsilon+45} \\
& \hat{u}_{\dot{\tau}_{2}}=u_{\dot{\tau}_{2}}\left(\frac{3 \varepsilon+27}{\varepsilon+45}, \frac{36}{\varepsilon+45}, \frac{72}{\varepsilon+45}\right)=\frac{3\left(17 \varepsilon^{2}+1746 \varepsilon+38,961\right)}{(\varepsilon+45)^{2}} \\
& \hat{u}_{\dot{\tau}_{2}}=u_{\dot{\tau}_{2}}\left(\frac{3 \varepsilon+27}{\varepsilon+45}, \frac{36}{\varepsilon+45}, \frac{72}{\varepsilon+45}\right)=\frac{9\left(7 \varepsilon^{2}+702 \varepsilon+15,399\right)}{(\varepsilon+45)^{2}}
\end{aligned}
$$


Then one can verify that for $\varepsilon \leq \frac{1}{2}$ there exist no Pareto improvements $\left(x_{1}, x_{2}, 3-x_{1}-x_{2}\right)$ over this utility tuple.

\section{Nudge}

One can interpret an increase in $\varepsilon$ as the result of an ex ante nudge, that sobers up self 1 at least partially, and makes it consider the possibility that its present bias need not disappear tomorrow (type $\ddot{\tau}_{2}$ has no present bias), but might rather persist (type $\dot{\tau}_{2}$ ) with probability $\varepsilon$.

Such a nudge, though, will decrease the well-being $\hat{u}_{\dot{t}_{1}}$ of self 1 at the no-intervention backward induction path, because

$$
\frac{d \hat{u}_{\dot{\tau}_{1}}}{d \varepsilon}=-\frac{324}{(\varepsilon+45)^{2}}<0 .
$$

Thus, even such an informational intervention, involving no coercion or incentives, is not Pareto improving.

\section{A.3.3 | Minimax intervention}

With the lack of Pareto-improvements, it may still be the case, though, that by disappointing some of the selves while cheering up others the parent can improve upon the average utility (3) this case to choosing a path that will minimize the maximal disappointment vis-a-vis (*****) across the three selves:

$$
\min _{x_{1}+x_{2}+x_{3}=3} \max _{i=1,2,3}\left[\hat{u}_{\dot{\tau}_{i}}-u_{i_{i}}\left(x_{1}, x_{2}, x_{3}\right)\right]
$$

The solution is $\left(\tilde{x}_{\dot{t}_{1}}, \tilde{x}_{\dot{\tau}_{2}}, \tilde{x}_{\dot{\tau}_{3}}\right)=(0.6734,0.9798,1.3468)$, with which $\dot{\tau}_{1}$ and $\dot{\tau}_{2}$ lose 0.0404 of their utility, each, but $\dot{\tau}_{3}$ gains 0.333 , overall leading to the higher average utility

$$
v\left(\tilde{x}_{\dot{t}_{1}}, \tilde{x}_{i_{2}}, \tilde{x}_{\dot{t}_{3}}\right)=57.537>v\left(\hat{x}_{\dot{\tau}_{1}}, \hat{x}_{\dot{t}_{2}}, \hat{x}_{\dot{t}_{3}}\right)
$$

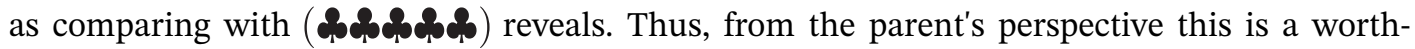
while intervention.

This intervention is in some sense even more "benign" than the informational nudge considered above, because it makes sure to smooth as much as possible the sobering pain of disillusionment across the different periods, rather than weighing its entire toll on the child in the first period only. 